
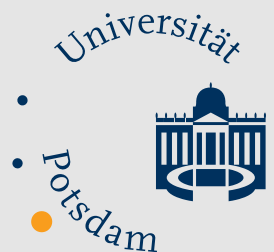

Humanwissenschaftliche Fakultät

Julia Schwind | Julia M. B. Neng | Florian Weck

\title{
Changes in Free Symptom Attributions in Hypochondriasis after Cognitive Therapy and Exposure Therapy
}

Suggested citation referring to the original publication:

Behavioural and Cognitive Psychotherapy 44 (2016)

DOI http://dx.doi.org/10.1017/S1352465816000163

ISSN (print) 1352-4658 - 0141-3473

ISSN (online) 1469-1833

Postprint archived at the Institutional Repository of the Potsdam University in:

Postprints der Universität Potsdam

Humanwissenschaftliche Reihe ; 457

ISSN 1866-8364

http://nbn-resolving.de/urn:nbn:de:kobv:517-opus4-414169 



\title{
Changes in Free Symptom Attributions in Hypochondriasis after Cognitive Therapy and Exposure Therapy
}

\author{
Julia Schwind and Julia M. B. Neng \\ Goethe University Frankfurt, Germany \\ Florian Weck \\ University of Potsdam, Germany
}

\begin{abstract}
Background: Cognitive-behavioural therapy can change dysfunctional symptom attributions in patients with hypochondriasis. Past research has used forced-choice answer formats, such as questionnaires, to assess these misattributions; however, with this approach, idiosyncratic attributions cannot be assessed. Free associations are an important complement to existing approaches that assess symptom attributions. Aims: With this study, we contribute to the current literature by using an open-response instrument to investigate changes in freely associated attributions after exposure therapy (ET) and cognitive therapy (CT) compared with a wait list (WL). Method: The current study is a re-examination of a formerly published randomized controlled trial (Weck, Neng, Richtberg, Jakob and Stangier, 2015) that investigated the effectiveness of CT and ET. Seventy-three patients with hypochondriasis were randomly assigned to CT, ET or a WL, and completed a 12-week treatment (or waiting period). Before and after the treatment or waiting period, patients completed an Attribution task in which they had to spontaneously attribute nine common bodily sensations to possible causes in an open-response format. Results: Compared with the WL, both CT and ET reduced the frequency of somatic attributions regarding severe diseases (CT: Hedges's $g=1.12$; ET: Hedges's $g=1.03$ ) and increased the frequency of normalizing attributions (CT: Hedges's $g=1.17$; ET: Hedges's $g=1.24)$. Only CT changed the attributions regarding moderate diseases (Hedges's $g=0.69$ ). Changes in somatic attributions regarding mild diseases and psychological attributions were not observed. Conclusions: Both CT and ET are effective for treating freely associated misattributions in patients with hypochondriasis. This study supplements research that used a forced-choice assessment.
\end{abstract}

Keywords: Attribution, hypochondriasis, free association, cognitive-behavioural therapy.

\section{Introduction}

According to the fourth edition of the Diagnostic and Statistical Manual of Mental Disorders (DSM-IV; American Psychiatric Association, 2000), one of the main characteristics of hypochondriasis is "the person's misinterpretation of bodily symptoms". With the newly

Correspondence to Julia Schwind, Department of Clinical Psychology and Psychotherapy, Varrentrappstraße 40-42, 60486 Frankfurt, Germany. E-mail: schwind@psych.uni-frankfurt.de 
published DSM-5 (American Psychiatric Association, 2013), hypochondriasis can now be divided into two different diagnoses: "somatic symptom disorder" and "illness anxiety disorder". In contrast to illness anxiety disorder, somatic symptom disorder includes the presence of somatic symptoms as a key diagnostic element. Because the presence of one or more somatic symptoms is not required in hypochondriasis, illness anxiety disorder is more consistent with the DSM-IV diagnosis for hypochondriasis. Increased sensitivity to concerns regarding health also remains a key diagnostic element of illness anxiety disorder. In addition, according to the cognitive-behavioural model that was proposed by Warwick and Salkovskis (1990), the misinterpretation of bodily symptoms as signs of a severe illness constitutes a key factor in the exacerbation and maintenance of hypochondriasis. This dysfunctional symptom attribution leads to a vicious circle of fear, increased bodily vigilance, an enhanced perception of bodily sensations and engagement in safety behaviour (Abramowitz, Deacon and Valentiner, 2007; Marcus, Gurley, Marchi and Bauer, 2007; Warwick and Salkovskis, 1990).

Empirical evidence for the importance of symptom attributions for hypochondriacal fears is strong (see, for example, Bailer et al., 2013; Barsky et al., 2001; Barsky, Coeytaux, Sarnie and Cleary, 1993; Fergus, 2014; Hadjistavropoulos, 1998; Haenen, Jong, Schmidt, Stevens and Visser, 2000; Haenen, Schmidt, Schoenmakers and van den Hout, 1998; Hitchcock and Mathews, 1992; MacLeod, Haynes and Sensky, 1998; Marcus, 1999; Marcus and Church, 2003; Rief, Hiller and Margraf, 1998; Schmidt, Witthöft, Kornadt, Rist and Bailer, 2013; Weck and Höfling, 2015, Weck, Neng, Richtberg and Stangier, 2012a, 2012b). Assessment of symptom attributions, however, can be conducted by using various approaches. Most commonly, questionnaires with forced-choice, closedended answer formats are used. In addition, more experimental approaches, such as cardsorting techniques, or implicit assessment strategies, such as the newly developed IATHypochondriasis (Weck and Höfling, 2015), can be used. Another important approach to assessing symptom attributions is free associations. Compared with forced-choice answer formats, free associations offer many advantages. Because free associations are not restricted and patients can freely specify every symptom that comes to mind, free associations are closer to the patients' reality, which increases the validity of the measurement. Especially in the field of hypochondriasis, it is important to detail individual perspectives, because patient attributions may vary over time. This variation may, for example, be strongly influenced by current media coverage of special diseases (e.g. amyotrophic lateral sclerosis, ALS). If these attributions are not assessed, important information concerning a patient's symptoms would be neglected. Another important advantage of free associations is the possibility to differentiate somatic attributions regarding different degrees of illness severity. Thus, free associations allow additional information on symptom attributions to be collected (Neng and Weck, 2015).

In the field of somatoform disorders in general, a few prior studies have attempted to overcome the disadvantage of forced-choice instruments by using free associations. Misattributions, for example, have been assessed in interviews (Groben and Hausteiner, 2011; Hiller et al., 2010; Martin et al., 2007). With regard to hypochondriasis, however, empirical evidence of dysfunctional symptom attributions assessed through free associations is scarce. One method to freely assess symptom attributions is the Attribution Task (Sensky, Haynes, Rigby and MacLeod, 1998). In this task, different common bodily sensations are presented (e.g. prolonged headache) to participants, who then have one minute per 
symptom to spontaneously attribute possible causes. Neng and Weck (2015) reported one notable study in which the Attribution Task is used to compare symptom attributions among patients with hypochondriasis, patients with anxiety disorders and healthy controls. In this study, two independent blind raters classified the given attributions into different categories: normalizing, somatic (diseases) and psychological. By using this instrument, Neng and Weck (2015) showed that hypochondriasis is associated with a disorder-specific attribution style that connects somatic symptoms primarily with moderate (e.g. migraine) and severe (e.g. cancer) diseases. Furthermore, patients with hypochondriasis used normalizing attributions (e.g. not enough sleep) less often than patients with diagnosed anxiety disorders and healthy controls. No differences in the frequency of psychological attributions (e.g. anxiety) and somatic attributions concerning mild diseases (e.g. having a cold) were found.

The hypochondriasis-specific attribution style that is reported by Neng and Weck (2015) raises the question whether cognitive-behavioural therapy (CBT) can change freely associated attributions regarding normalizing, somatic and psychological explanations. In general, CBT has been demonstrated to be highly effective in the treatment of hypochondriasis (Olatunji et al., 2014). However, until now, the empirical evidence that CBT can change dysfunctional symptom attributions is based on forced-choice instruments only. For instance, Weck, Neng, Schwind and Höfling (2015) used the Symptoms Outcome Scale (Marcus, 1999) and the Health Norms Sorting Task (Barsky et al., 1993), a card-sorting technique, to measure changes in symptom attributions in hypochondriasis. ${ }^{1}$ They compared exposure therapy (ET) and cognitive therapy (CT) with assignment to a waitlist (WL), and the active treatment included 12 weekly sessions that occurred over a 3-month period. The results showed that compared with the WL group, both the ET and CT groups significantly changed their dysfunctional symptom evaluations after treatment. No differences between the CT and ET groups were found. Moreover, changes in health-related beliefs mediated the relation between the treatment condition and reductions in health anxiety. This finding underlines the importance of modifying health-related beliefs to facilitate effective treatment.

It is unclear, however, whether the results of Weck, Neng and Schwind et al. (2015) are applicable to free associations. Investigating free associations in a longitudinal design therefore constitutes a valuable contribution to the literature. Such an investigation would also allow the severity of somatic attributions to be considered. Such research is necessary to elucidate the influence of CBT on somatic attributions with different degrees of severity. With the present study, we therefore aim to extend the current literature by using the Attribution Task. Accordingly, we re-examined the study by Weck, Neng, Richtberg et al. (2015) and analysed the changes in free symptom attributions in patients with hypochondriasis after CBT. We used a longitudinal design and compared CT and ET patients with a WL control group. We hypothesized that compared with WL, both ET and CT would lead to significant reductions in free attributions regarding serious and moderate diseases (Hypothesis 1a) and significant increases in normalizing attributions (Hypothesis 1b). Based on the results of Neng and Weck (2015), we also expected to find no changes in attributions regarding mild diseases (Hypothesis 2a) or psychological attributions (Hypothesis 2b). Furthermore, in line with the recent findings of Weck, Neng, Schwind et al. (2015), we

\footnotetext{
${ }^{1}$ This study, similar to the present study, is based on the RCT by Weck, Neng, Richtberg, Jakob and Stangier (2015).
} 
hypothesized that changes in attributions would not differ between the CT and ET groups (Hypothesis 3).

\section{Method}

The current study was based on a RCT that compared CT, ET, and assignment to a WL (see Weck, Neng, Richtberg et al., 2015 for a detailed description). Eighty-four patients with a primary diagnosis of hypochondriasis were randomized to either CT, ET or a WL. After completing the WL condition, the patients were also randomized to either CT or ET. The results showed that compared with the WL, both CT and ET proved effective in both primary (Yale-Brown Obsessive Compulsive Scale for Hypochondriasis; H-YBOCS; Weck, Gropalis, Neng, and Witthöft, 2013) and secondary (several standardized self-report measures that evaluated hypochondriacal characteristics and general psychopathology) outcome measures. No differences were detected for CT and ET. The current study included 73 patients who completed the Attribution task at pre and posttreatment (completer sample).

\section{Participants}

All 73 participants met the DSM-IV criteria of hypochondriasis according to the Structured Clinical Interview for DSM-IV (SCID; First, Spitzer, Gibbon and Williams, 1997), which was conducted by experienced diagnosticians who were trained in a 2-day SCID workshop. The inclusion criteria were a primary diagnosis of hypochondriasis. The exclusion criteria were age under 18 or over 67 years, deficiencies in the German language, a major medical illness (e.g. cancer), acute suicidality, and a clinical diagnosis of substance addiction, schizophrenia or schizoaffective disorder, or bipolar disorder according to the SCID. Relative to the WL, both ET and CT demonstrated their efficacy in reducing hypochondriacal cognitions and behaviours (primary outcome measures) and several psychopathological characteristics (secondary outcome measures). Furthermore, the completer analysis revealed no differences between CT and ET on the primary and secondary outcome measures. However, safety behaviour was reduced to a greater extent after ET (see Weck, Neng, Richtberg et al., 2015). Figure 1 provides an overview of the participant recruitment. The 73 patients ranged from 18 to 67 years old, with an average age of 40.07 years $(S D=$ 11.65). Forty-three of the participants were female (58.9\%). Fifty patients $(68.5 \%)$ had the qualifications for university entrance. The majority of the patients $(53.4 \%)$ had at least one comorbid disorder (comorbid anxiety disorder: 38.4\%, comorbid affective disorder: $19.2 \%)$. The most feared disease was cancer (69.9\%). No significant differences were found between the three groups (ET, CT, and WL) regarding participants' sociodemographic and psychopathological characteristics, including comorbid diagnoses and most feared diseases (each $p>.05)$.

\section{Measures}

Attribution task. The Attribution task is based on the work of Sensky et al. (1998). We used nine common bodily sensations (prolonged headache, getting dizzy, feeling fatigued, hand trembling, having trouble sleeping, upset stomach, losing appetite, difficulties catching breath, and numbness or tingling in hands or feet) from the SIQ (Robbins and Kirmayer, 


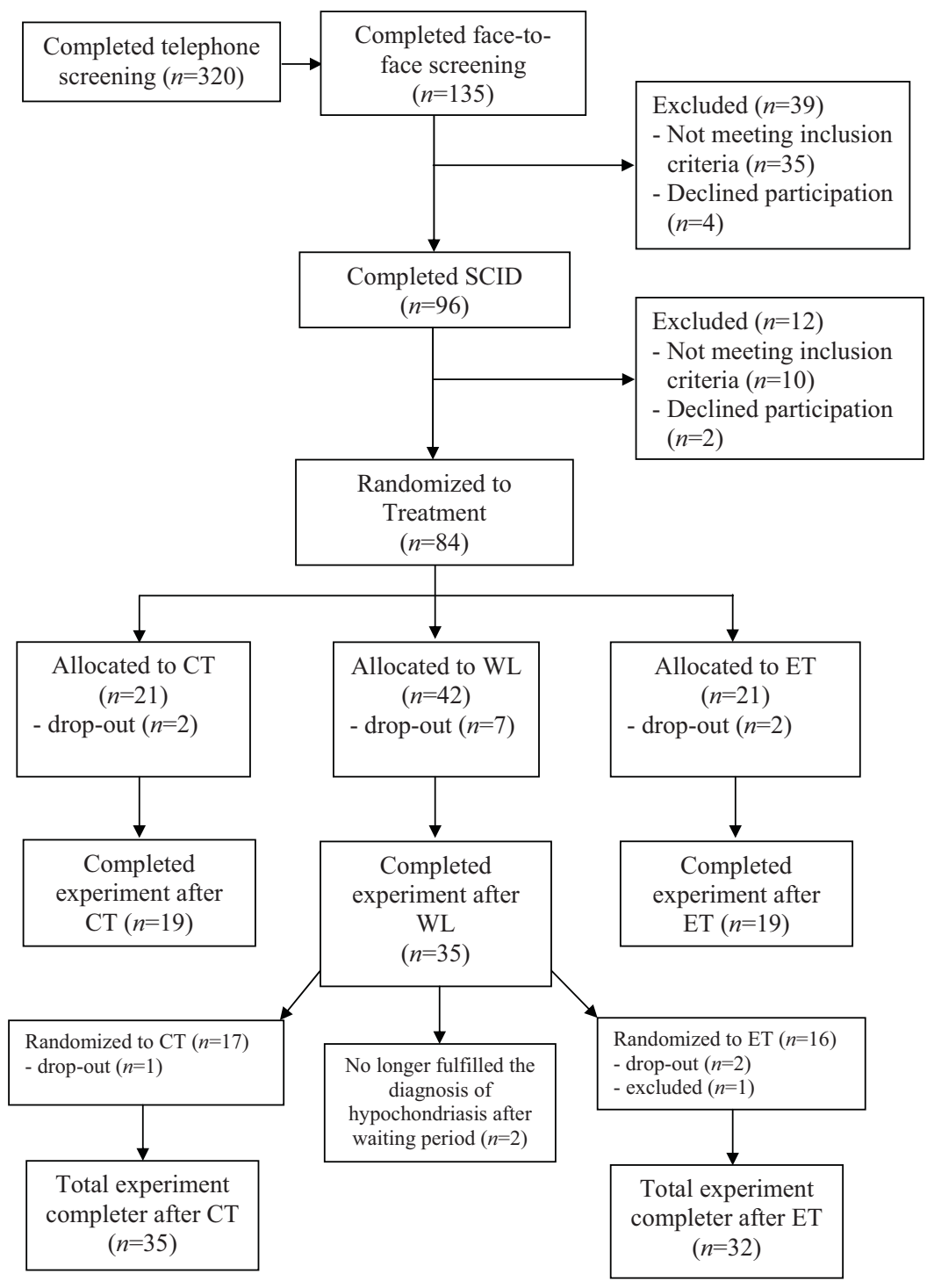

Figure 1. Participant flow chart

Notes: $\mathrm{CT}=$ cognitive therapy; $\mathrm{WL}=$ wait list; $\mathrm{ET}=$ exposure therapy; SCID: Structured Clinical Interview for the Diagnostic and Statistical Manual of Mental Disorders $\left(4^{\text {th }}\right.$ edn).

1991). Each symptom was printed on a separate sheet of paper and presented to the participant with the instruction to imagine that the symptom would affect her or him at the moment. Symptoms were always presented in the same sequence. The participants were told to write down any explanations they would have below the presented symptom. They had 1 minute for each symptom, and the experimenter then gave the instruction to turn the page for the 
next symptom, thus standardizing the procedure and ensuring that the participants spent the same amount of time on each symptom. In doing so, the task should be thoroughly processed, preventing early termination of the task, e.g. from fear-related avoidance.

The provided attributions were classified into three categories: normalizing (e.g. insufficient intake of food or fluids, fatigue or overexertion), somatic (diseases) and psychological (e.g. nervousness or excitement). Based on the work by Weck, Neng, Göller and MüllerMarbach (2014), we further segmented illnesses into the categories mild, moderate and severe depending on the severity of the illness. Illnesses without lasting impairment were categorized as mild (e.g. a cold), whereas illnesses associated with moderate impairment were categorized as moderate (e.g. migraine, asthma). Finally, chronic illnesses with massive impairment and potentially life threatening illnesses were categorized as severe (e.g. cancer, HIV, myocardial infection). The interrater reliability of two independent raters for the entire classification was found to be $\kappa=.90, p<.001,95 \%$ CI $(0.89,0.91)$, which is almost in perfect agreement with and at the same level as the findings of Neng and Weck $(2015 ; \kappa=$ $.90, p<.001,95 \% \mathrm{CI}(0.89,0.91)$. The raters were blinded with respect to the treatment conditions.

\section{Treatment}

For a detailed description of the interventions, see Weck, Neng, Richtberg et al. (2015). In both CT and ET, the first session was used for a detailed psychoeducation regarding the clinical picture of hypochondriasis. Depending on the condition, either the role of selective attention (CT) or the avoidance and safety behaviour (ET) was presented. In CT, sessions 2 and 3 were used to demonstrate the importance of selective attention to bodily sensations through behavioural experiments, which were followed by attentionrelated exercises designed to reduce body-focused attention. Sessions 4 to 11 focused on the reduction of dysfunctional health-related automatic beliefs. These interventions mainly involved searching for alternative, non-threatening explanations for bodily symptoms and the evaluation of these explanations. For this reason, cognitive restructuring, behavioural experiments, and imagery rescripting for the modification of intrusive images were conducted.

ET did not include any explicit cognitive interventions; rather, it focused on exposing the patient to stimuli that are relevant for health anxieties (e.g. documentaries regarding diseases), reducing avoidance behaviour (e.g. cemeteries) and safety behaviour (e.g. reassurance seeking on the internet). Here, sessions 2 and 3 focused on the reduction of patients' safety behaviour step-by-step. Sessions 5 to 11 focused on the implementation of the treatment rationale for exposure and multiple exercises of exposure (i.e. interoceptive exposure, exposure in vivo, and exposure in sensu). A more detailed description of ET has been provided in previous research (Weck, Ritter and Stangier, 2012). In both conditions, session 12 focused on relapse prevention and the discussion on how the techniques can be established for the time after treatment. The purity index (Luborky, McLellan, Woody, O'Brian and Auerbach, 1985), which reflects the ratio of the intended interventions to the presence of intended plus unintended interventions, indicated very high therapist adherence in both treatment conditions (CT: 0.94, SD: 0.10; ET: 0.96; SD: 0.07; see Weck, Neng, Richtberg et al., 2015 for a more detailed description). The waiting period for the WL also lasted 12 weeks and did not include any treatment. 


\section{Procedure}

After initial contact, the patients were randomly assigned to CT, ET, or the WL, and they completed the Attribution task both before the treatment/waiting period started and after the treatment/waiting period ended. After the waiting period, the participants in the WL were randomized to $\mathrm{CT}$ or ET, and they also completed the Attribution task after the treatment ended (see Figure 1).

\section{Statistical analyses}

The possible differences between the groups concerning pretreatment sociodemographic characteristics, comorbid diagnoses, and most feared diseases were analysed with $\chi^{2}$ tests for the categorical variables or a series of analyses of variance (ANOVAs) for the continuous variables.

Interrater agreement in the Attribution task was determined by using Cohen's $\kappa$. The relative frequencies were summed for all symptoms and then analysed as described below. For each attribution category and symptom, we computed the relative frequency of use. We thus divided the number of attributions in each category by the total number of attributions per symptom. In this way, we were able to avoid distortions due to different numbers of freely associated attributions.

A two-stage strategy was used for the data analyses of treatment effects. In the first stage, analyses of covariance (ANCOVAs) using pretreatment (or pre-waiting period) scores as a covariate, group (CT, ET, or WL) as the independent variable, and the posttreatment (or postwaiting period) scores as the dependent variable were conducted. Post-hoc comparisons were used to investigate differences between the three conditions.

In the second stage, the analysis also included participants who were initially randomized to the WL and subsequently assigned to ET or CT, and ANCOVAs using pretreatment scores as a covariate, group (CT or ET) as the independent variable, and the posttreatment scores as the dependent variable were again conducted.

Effect sizes were obtained via Hedges's $g$. All reported $p$ values were two tailed, and a conventional $\alpha$ significance level of .05 was used.

\section{Results}

Comparison between cognitive therapy, exposure therapy, and wait list (first step of the analysis)

ANCOVAs revealed a significant main effect of treatment group on the frequency of the use of normalizing attributions and attributions regarding moderate and severe diseases (see Table 1). Post-hoc pairwise comparisons of the posttreatment scores of the frequency of the use of normalizing attributions revealed significant differences between CT and WL ( $p<$ .001 , Hedges's $g=1.17)$ and between ET and WL $(p<.001$, Hedges's $g=1.24)$ but not between CT and ET ( $p=.36$, Hedges's $g=0.05$ ). Post hoc pairwise comparisons of the posttreatment scores of the frequency of the use of attributions regarding moderate diseases revealed significant differences between CT and WL $(p<.01$, Hedges's $g=0.69)$ but not between ET and WL ( $p=.10$, Hedges's $g=0.52)$ or between CT and ET ( $p=.33$, Hedges's $g=0.17$ ). Post hoc pairwise comparisons of the post-treatment frequency of the use of 
Table 1. Mean scores and standard deviations of the relative frequency of use of the categories in the Attribution task for the completer sample

\begin{tabular}{|c|c|c|c|c|c|c|c|c|}
\hline \multirow[b]{2}{*}{ Measures } & \multicolumn{2}{|c|}{$\begin{array}{c}\text { Cognitive therapy } \\
M(S D) \\
n=19\end{array}$} & \multicolumn{2}{|c|}{$\begin{array}{l}\text { Exposure therapy } \\
\begin{array}{c}M(S D) \\
n=19\end{array}\end{array}$} & \multicolumn{2}{|c|}{$\begin{array}{l}\text { Waiting list } \\
\begin{array}{l}M(S D) \\
n=35\end{array}\end{array}$} & \multicolumn{2}{|c|}{ ANCOVA } \\
\hline & Pre & Post & Pre & Post & Pre & Post & $F$-value & Group differences \\
\hline Normalizing & $0.35(0.14)$ & $0.50(0.18)$ & $0.32(0.16)$ & $0.51(0.17)$ & $0.28(0.15)$ & $0.31(0.15)$ & $13.29^{* * *}$ & CT-WL, ET-WL \\
\hline Somatic - mild disease & $0.07(0.05)$ & $0.07(0.06)$ & $0.06(0.05)$ & $0.08(0.06)$ & $0.06(0.05)$ & $0.07(0.06)$ & $0.10^{\text {n.s. }}$ & - \\
\hline Somatic-moderate disease & $0.14(0.07)$ & $0.05(0.06)$ & $0.10(0.09)$ & $0.06(0.06)$ & $0.11(0.08)$ & $0.10(0.07)$ & $4.68^{*}$ & CT-WL \\
\hline Somatic - severe disease & $0.17(0.13)$ & $0.06(0.08)$ & $0.21(0.15)$ & $0.07(0.12)$ & $0.28(0.20)$ & $0.24(0.18)$ & $12.52^{* * *}$ & CT-WL, ET-WL \\
\hline Psychological & $0.26(0.13)$ & $0.29(0.13)$ & $0.27(0.13)$ & $0.26(0.09)$ & $0.25(0.14)$ & $0.28(0.14)$ & $0.56^{\text {n.s. }}$ & - \\
\hline
\end{tabular}

Notes: ANCOVA: analyses of covariance; pre $=$ pretreatment; post $=$ posttreatment $;{ }^{*} p<.05 ;{ }^{* *} p<.01 ;{ }^{* * *} p<.001$. 
Table 2. Mean scores and standard deviations of the relative frequency of use of the categories in the Attribution task at pre and posttreatment for the cognitive therapy and exposure therapy groups

\begin{tabular}{|c|c|c|c|c|c|}
\hline \multirow[b]{2}{*}{ Measures } & \multicolumn{2}{|c|}{$\begin{array}{c}\text { Cognitive therapy } \\
\begin{array}{c}M(S D) \\
n=35\end{array}\end{array}$} & \multicolumn{2}{|c|}{$\begin{array}{c}\text { Exposure therapy } \\
\qquad \begin{array}{c}M(S D) \\
n=32\end{array}\end{array}$} & \multirow{2}{*}{$\begin{array}{l}\text { ANCOVA } \\
F \text {-value }\end{array}$} \\
\hline & Pre & Post & Pre & Post & \\
\hline Normalizing & $0.33(0.16)$ & $0.46(0.17)$ & $0.32(0.15)$ & $0.49(0.17)$ & $1.41^{\text {n.s. }}$ \\
\hline Somatic - mild disease & $0.08(0.06)$ & $0.07(0.57)$ & $0.06(0.05)$ & $0.08(0.06)$ & $0.87^{\text {n.s. }}$ \\
\hline Somatic - moderate disease & $0.12(0.07)$ & $0.07(0.06)$ & $0.10(0.08)$ & $0.06(0.06)$ & $0.07^{\text {n.s. }}$ \\
\hline Somatic - severe disease & $0.19(0.16)$ & $0.09(0.13)$ & $0.23(0.16)$ & $0.08(0.13)$ & $1.16^{\text {n.s. }}$ \\
\hline Psychological & $0.27(0.14)$ & $0.29(0.14)$ & $0.27(0.13)$ & $0.25(0.12)$ & $2.99^{\text {n.s. }}$ \\
\hline
\end{tabular}

Notes: ANCOVA: analyses of covariance; pre $=$ pretreatment; post $=$ posttreatment.

attributions regarding severe diseases revealed significant differences between CT and WL $(p<.001$, Hedges's $g=1.12)$ and between ET and WL $(p<.001$, Hedges's $g=1.03)$ but not between CT and ET ( $p=.38$, Hedges's $g=0.05$ ). No differences in the frequency of the use of psychological attributions and attributions regarding mild diseases were found (each $p>.05$ ).

Comparison between cognitive therapy and exposure therapy for the outcomes at posttreatment (second step of the analysis)

The results of this analysis, as well as the mean scores and standard deviations of the relative frequencies of use of the categories in the Attribution task at pre and posttreatment for all participants in the CT and ET groups who completed the Attribution task, are shown in Table 2. ANCOVAs showed no significant main effect of condition (each $p>.05$ ).

\section{Discussion}

The present study expands the current literature by examining changes in symptom attributions in patients with hypochondriasis after CT and ET with an open-response format. The results indicate that in comparison with a WL, both CT and ET can significantly reduce the frequency of somatic attributions regarding severe diseases (Hypothesis 1a) and significant increase the frequency of normalizing attributions (Hypothesis 1b). Contrary to our expectations, only CT was able to change the somatic attributions regarding moderate diseases; however, ET showed a trend towards a significant change (Hypothesis 1a). Consistent with our expectations, the frequencies of mild somatic attributions (Hypothesis 2a) and psychological attributions (Hypothesis $2 b$ ) did not change after therapy. Consistent with previous analyses of the sample (Weck, Neng, Schwind et al., 2015), the direct comparison of $\mathrm{CT}$ and ET also revealed no differences between the conditions in the open-response format (Hypothesis 3).

Previous research was only able to show that CT and ET can change the prescribed symptom attributions, which were assessed with only forced-choice instruments (Weck, Neng, Schwind et al., 2015). Our study, however, is the first to show that changes in 
symptom attributions after CT and ET can also be shown through free associations, which enables the evaluation of more individual perspectives of the patients. The specific pattern of attributional changes that is observed in this study corresponds to the hypochondriasis-specific attribution style that was found by Neng and Weck (2015), and our results show that these particular patterns can be changed with psychotherapy. Future research should also analyse the combined effects of cognitive and exposure-based interventions on free symptom attributions in hypochondriasis to account for the possible incremental effects of a combination of CT and ET.

By using the Attribution Task, we were able to assess symptom attributions in an openresponse format. This format enabled the assessment of idiosyncratic attribution and the investigation of several gradations of symptom severity by using the normalizing, somatic, and psychological symptom categories. Furthermore, we obtained almost perfect interrater reliability, which indicates that the Attribution Task is a suitable instrument for the specific near-patient assessment of free associations and their changes after CBT. Compared with previously used forced-choice instruments, free associations offer a valuable contribution to the literature and previous analyses of the used sample (see Weck, Neng, Schwind et al., 2015). Therefore, future studies regarding symptom attributions in hypochondriasis may benefit from using the Attribution Task for the assessment of more individual symptom attributions. Moreover, the Attribution Task may provide additional information in clinical practice because it can facilitate a systematic and more exhaustive assessment of dysfunctional symptom attributions than an oral exploration. A better assessment may facilitate, for example, more tailored interventions. The Attribution Task therefore expands the assessment possibilities for therapists.

In our study we found that not only CT but also ET can reduce the frequency of severe somatic attributions and increase the frequency of normalizing attributions, although ET does not include any cognitive interventions (Hypotheses 1a and 1b). Thus, although ET does not focus on explicitly changing misattributions, it is nevertheless effective at changing them. This finding supports the results of previous analyses of the same sample by Weck, Neng, Schwind et al. (2015) who used a forced-choice task. In this study, the need for cognitive interventions for hypochondriasis has already been challenged. However, ET seems to be able to disconfirm misattributions by violating expectations without explicitly targeting them with cognitive techniques. Through experiential evidence, ET can thus cause the same cognitive changes as CT, as proposed in the inhibitory learning model (see, for example, Arch and Abramowitz, 2014; Bouton, 1993; Bouton, Woods, Moody, Sunsay and García-Gutiérrez, 2006; Craske et al., 2008; Craske, Treanor, Conway, Zbozinek and Vervliet, 2014; Vervliet, Craske and Hermans, 2013). In this process, non-danger based associations between the conditioned and unconditioned stimulus are formed ("Dizziness is not dangerous and may occur"), and fear tolerance ("Fear is tolerable") is increased; then, the non-danger based associations compete with original danger based associations ("Dizziness is dangerous and a sign of a brain tumour").

Interestingly, ET does not seem to be equally effective in changing various types of hypochondriasis-specific attributions. We further found that compared with assignment to a WL, only CT can change the attributions regarding moderate diseases; however, we observed a trend towards a significant change in moderate somatic symptoms through ET. Thus, we may have been able to find a significant effect with a larger sample size. Nevertheless, compared with ET, CT appears to have a broader spectrum of efficacy to change the somatic attributions 
of both moderate and severe diseases. Previous findings have shown that changes in healthrelated beliefs mediate the relation between treatment condition and health anxiety (Weck, Neng, Schwind et al., 2015), which emphasizes the importance of CT in the treatment of hypochondriasis. Therefore, future studies should further compare the efficacy of CT and ET on different degrees of severity of symptom attributions.

An explanation for the broader efficacy of CT compared with ET may lie in the treatment rationale itself. CT may simply offer more space to discuss a very broad range of bodily symptoms that focus not only on severe diseases but also on moderate somatic attributions. To facilitate Socratic reasoning regarding the origins of bodily symptoms, patients also receive detailed information in the form of psychoeducation concerning the autonomic nervous system and bodily sensations in general. In contrast, ET explicitly confronts the patient with her or his worst personal fears; thus, ET is more tailored and more often addresses severe somatic attributions. However, one may argue that somatic attributions regarding severe diseases are more relevant to hypochondriasis. This explanation is consistent with the definition of hypochondriasis in the DSM-IV (American Psychiatric Association, 2000), which includes a preoccupation with having or acquiring a serious (not moderate) illness. Furthermore, regarding somatic attributions, Neng and Weck (2015) showed that patients with hypochondriasis differed the most from patients with anxiety disorders $(d=1.61)$ and healthy controls $(d=1.91)$ in severe diseases. However, Neng and Weck also found significant differences concerning moderate diseases, which indicates that patients with hypochondriasis also show specific deviations regarding this degree of severity $(d=0.63$ compared with patients with anxiety disorders, $d=1.13$ compared with healthy controls).

In the second step of the analysis with all treated patients, however, our results confirmed that the differences between CT and ET are not significant. Considering the clinical implications of our findings, we therefore provide evidence that both CT and ET are appropriate for treating misattributions in patients with hypochondriasis. Therefore, clinicians working primarily with exposure can also adequately address attributional patterns.

\section{Limitations}

Some limitations of the current study should be noted. First, our sample comprises patients with hypochondriasis who were willing to receive psychological treatment. Often, however, patients with hypochondriasis remain in purely medical settings; thus, we may have examined a subgroup of patients with hypochondriasis who show particular openness to psychological treatment or who have a less rigid dysfunctional health-belief system. Second, although the Attribution task has many advantages over common questionnaires, the presentation of the symptoms is still prescribed. Patients may thus suffer from completely different symptoms than the presented symptoms. However, in hypochondriasis, we can assume that the disorderspecific, dysfunctional cognitive pattern can also be reflected in the evaluations of other symptoms.

In summary, our results expand current knowledge by showing that both CT and ET are effective for changing freely associated misattributions in patients with hypochondriasis. We recommend a detailed assessment of individual symptom attributions before therapy. Future research should also integrate a more differential perspective. More profound knowledge of patient variables that may moderate the effect of CT and ET on symptom attributions would 
help to inform the selection of the most promising treatment approach, possibly increasing response rates.

\section{Acknowledgements}

This research was supported by grants WE 4654/2-1 and WE 4654/2-3 from the German Research Foundation. We want to thank MSc Margit Kiechle for her help with the data collection.

Ethical standards: The authors assert that all procedures that contribute to this work comply with the ethical standards of the relevant national and institutional committees on human experimentation and with the Helsinki Declaration of 1975 and its most recent revision. The authors also assert that all procedures that contribute to this work comply with the ethical standards of the relevant national and institutional guides on the care and use of laboratory animals.

Conflict of interest: The authors have no conflicts of interest with respect to this publication.

\section{References}

Abramowitz, J. S., Deacon, B. J. and Valentiner, D. P. (2007). The Short Health Anxiety Inventory: psychometric properties and construct validity in a non-clinical sample. Cognitive Therapy and Research, 31, 871-883. doi:10.1007/s10608-006-9058-1

American Psychiatric Association (2000). Diagnostic and Statistical Manual (4th edn). Washington, DC: APA.

American Psychiatric Association (2013). Diagnostic and Statistical Manual (5th edn). Washington, DC: APA.

Arch, J. J. and Abramowitz, J. S. (2014). Exposure therapy for obsessive-compulsive disorder: an optimizing inhibitory learning approach. Journal of Obsessive-Compulsive and Related Disorders. doi:10.1016/j.jocrd.2014.12.002

Bailer, J., Müller, T., Witthöft, M., Diener, C., Mier, D., Ofer, J., et al. (2013). Symptomattributionsstile bei Hypochondrie. Psychotherapeut, 58, 552-559. doi:10.1007/s00278-013-1014-4

Barsky, A. J., Ahern, D. K., Duff Bailey, E., Saintfort, R., Liu, E. B. and Peekna, H. M. (2001). Hypochondriacal patients' appraisal of health and physical risks. American Journal of Psychiatry, 158, 783-787.

Barsky, A. J., Coeytaux, R. R., Sarnie, M. K. and Cleary, P. D. (1993). Hypochondriacal patients beliefs about good health. American Journal of Psychiatry, 150, 1085-1089.

Bouton, M. E. (1993). Context, time, and memory retrieval in the interference paradigms of Pavlovian learning. Psychological Bulletin, 114, 80-99. doi:10.1037/0033-2909.114.1.80

Bouton, M. E., Woods, A. M., Moody, E. W., Sunsay, C. and García-Gutiérrez, A. (2006). Counteracting the context-dependence of extinction: relapse and tests of some relapse prevention methods. In M. G. Craske, D. Hermans and D. Vansteenwegen (Eds.), Fear and Learning: from basic processes to clinical implications (pp. 175-196). Washington, DC: American Psychological Association.

Craske, M. G., Kircanski, K., Zelikowsky, M., Mystkowski, J., Chowdhury, N. and Baker, A. (2008). Optimizing inhibitory learning during exposure therapy. Behaviour Research and Therapy, 46, 5-27. doi:10.1016/j.brat.2007.10.003 
Craske, M. G., Treanor, M., Conway, C. C., Zbozinek, T. and Vervliet, B. (2014). Maximizing exposure therapy: an inhibitory learning approach. Behaviour Research and Therapy, 58, 10-23. doi:10.1016/j.brat.2014.04.006

Fergus, T. A. (2014). Health-related dysfunctional beliefs and health anxiety: further evidence of cognitive specificity. Journal of Clinical Psychology, 70, 248-259. doi:10.1002/jclp.22012

First, M. B., Spitzer, R. L., Gibbon, M. and Williams, K. B. W. (1997). Structural Clinical Interview for DSM-IV Axis I Disorder (SCID-I). Washington DC: American Psychiatric Press.

Groben, S. and Hausteiner, C. (2011). Somatoform disorders and causal attributions in patients with suspected allergies: do somatic causal attributions matter? Journal of Psychosomatic Research, 70, 229-238. doi:10.1016/j.jpsychores.2010.09.002

Hadjistavropoulos, H. (1998). Cognitive and behavioral responses to illness information: the role of health anxiety. Behaviour Research and Therapy, 36, 149-164. doi:10.1016/S0005-7967(98)00014-X

Haenen, M.-A., Jong, P. J. de, Schmidt, A., Stevens, S. and Visser, L. (2000). Hypochondriacs' estimation of negative outcomes: domain-specificity and responsiveness to reassuring and alarming information. Behaviour Research and Therapy, 38, 819-833. doi:10.1016/S0005-7967(99)00128-X

Haenen, M.-A., Schmidt, A., Schoenmakers, M. and van den Hout, M.A. (1998). Quantitative and qualitative aspects of cancer knowledge: comparing hypochondriacal subjects and healthy controls. Psychology and Health, 13, 1005-1014.

Hiller, W., Cebulla, M., Korn, H.-J., Leibbrand, R., Röers, B. and Nilges, P. (2010). Causal symptom attributions in somatoform disorder and chronic pain. Journal of Psychosomatic Research, 68, 9-19. doi:10.1016/j.jpsychores.2009.06.011

Hitchcock, P. and Mathews, A. (1992). Interpretation of bodily symptoms in hypochondriasis. Behaviour Research and Therapy, 30, 223-234. doi:10.1016/0005-7967(92)90068-R

Luborsky, L., McLellan, A.T., Woody, G. E., O'Brien, C. P. and Auerbach, A. (1985). Therapist success and its determinants. Archives of General Psychiatry, 42, 602-611. doi:10.1001/archpsyc.1985.01790290084010

MacLeod, A. K., Haynes, C. and Sensky, T. (1998). Attributions about common bodily sensations: their associations with hypochondriasis and anxiety. Psychological Medicine, 28, 225-228.

Marcus, D. K. (1999). The cognitive-behavioral model of hypochondriasis: misinformation and triggers. Journal of Psychosomatic Research, 47, 79-91. doi:10.1016/S0022-3999(99)00008-2

Marcus, D. K. and Church, S. E. (2003). Are dysfunctional beliefs about illness unique to hypochondriasis? Journal of Psychosomatic Research, 54, 543-547. doi:10.1016/S0022-3999(02)00526-3

Marcus, D. K., Gurley, J. R., Marchi, M. M. and Bauer, C. (2007). Cognitive and perceptual variables in hypochondriasis and health anxiety: a systematic review. Clinical Psychology Review, 27, 127139. doi:10.1016/j.cpr.2006.09.003

Martin, A., Korn, H.-J., Cebulla, M., Saly, M., Fichter, M. M. and Hiller, W. (2007). Kausalattributionen von körperlichen Beschwerden bei somatoformen Störungen. Zeitschrift für Psychiatrie, Psychologie und Psychotherapie, 55, 31-41. doi:10.1024/1661-4747.55.1.31

Neng, J. M. B. and Weck, F. (2015). Attribution of somatic symptoms in hypochondriasis. Clinical Psychology and Psychotherapy, 22, 116-124. doi:10.1002/cpp.1871

Olatunji, B. O., Kauffman, B. Y., Meltzer, S., Davis, M. L., Smits, J. A. and Powers, M. B. (2014). Cognitive-behavioral therapy for hypochondriasis/health anxiety: a meta-analysis of treatment outcome and moderators. Behaviour Research and Therapy, 58, 65-74. doi:10.1016/j.brat.2014.05.002

Rief, W., Hiller, W. and Margraf, J. (1998). Cognitive aspects of hypochondriasis and the somatization syndrome. Journal of Abnormal Psychology, 107, 587-595.

Robbins, J. M. and Kirmayer, L. J. (1991).Attributions of common somatic symptoms. Psychological medicine, 21, 1029-1045. doi:10.1017/S0033291700030026

Schmidt, E., Witthöft, M., Kornadt, A., Rist, F. and Bailer, J. (2013). Negative automatic evaluation and better recognition of bodily symptom words in college students with elevated health anxiety. Cognitive Therapy and Research, 37, 1027-1040. doi:10.1007/s10608-013-9540-5 
Sensky, T., Haynes, C., Rigby, M. and MacLeod, A. (1998). Attribution von Körperempfindungen bei Patienten in der medizinischen Erstversorgung: Zusammenhänge mit der Häufigkeit von Arztbesuchen und Hypochondrie. Verhaltenstherapie, 8, 101-105.

Vervliet, B., Craske, M. G. and Hermans, D. (2013). Fear extinction and relapse: state of the art. Annual Review of Clinical Psychology, 9, 215-248. doi:10.1146/annurev-clinpsy-050212-185542

Warwick, H. M. and Salkovskis, P. M. (1990). Hypochondriasis. Behaviour Research and Therapy, 28, 105-117. doi:10.1016/0005-7967(90)90023-C

Weck, F., Gropalis, M., Neng, J. M. B. and Witthöft, M. (2013). The German version of the H-YBOCS for the assessment of hypochondriacal cognitions and behaviors: development, reliability and validity. International Journal of Behavioral Medicine, 20, 618-626. doi:10.1007/s12529-012-9276-8

Weck, F. and Höfling, V. (2015). Assessment of implicit health attitudes: a multitrait-multimethod approach and a comparison between patients with hypochondriasis and patients with anxiety disorders. Journal of Personality Assessment, 97, 55-65. doi:10.1080/00223891.2014.913253

Weck, F., Neng, J. M. B., Richtberg, S., Jakob, M. and Stangier, U. (2015). Cognitive therapy versus exposure therapy for hypochondriasis (health anxiety): a randomized controlled trial. Journal of Consulting and Clinical Psychology, 83, 665-676. doi:10.1037/ccp0000013

Weck, F., Neng, J. M. B., Richtberg, S. and Stangier, U. (2012a). Dysfunctional beliefs about symptoms and illness in patients with hypochondriasis. Psychosomatics, 53, 148-154.

Weck, F., Neng, J. M. B., Richtberg, S. and Stangier, U. (2012b). The restrictive concept of good health in patients with hypochondriasis. Journal of Anxiety Disorders, 26, 792-798. doi:10.1016/j.janxdis.2012.07.001

Weck, F., Neng, J. M. B., Schwind, J. and Höfling, V. (2015). Exposure therapy changes dysfunctional evaluations of somatic symptoms in patients with hypochondriasis (health anxiety): a randomized controlled trial. Journal of Anxiety Disorders, 34, 1-7.

Weck, F., Neng, J. M. B., Göller, K. and Müller-Marbach, A. M. (2014). Previous experiences with illness and traumatic experiences: a specific risk factor for hypochondriasis? Psychosomatics, 55(4), 362-371. doi:10.1016/j.psym.2013.10.005

Weck, F., Ritter, V. and Stangier, U. (2012). Variants of exposure in body dysmorphic disorder and hypochondriasis. In P. Neudeck and H.-U. Wittchen (Eds.), Exposure Therapy: rethinking the model - refining the methods (pp.217-244). New York: Springer. 